\title{
The benefits of steroids versus steroids plus antivirals for treatment of Bell's palsy: a meta-analysis
}

The authors of this Research paper have informed us that they misreported data from the paper by Engstrom et al that they used as part of their meta-analysis (BMJ 2009;339:b3354, doi:10.1136/bmj.b3354). In figure 2 the number of patients receiving steroids only at follow-up should have read 186 (not "180") and the number of patients receiving steroids plus antivirals at follow-up should have read 180 (not "186"). The authors state that this mix up in the denominator data changes the odds ratio (95\% confidence interval) for that study from 0.93 ( 0.49 to 1.77 ) to 1.66 ( 0.86 to 3.22 ) and add that an odds ratio greater than 1 favours steroids plus antivirals. They assert that this does not change the statistical significance of the result for that study but does change the pooled odds ratio from 1.50 ( 0.83 to 2.69 ) to 1.72 (1.02 to 2.88 ), with the lower limit of the confidence interval now just above 1 .

However, the authors would like to explain that these data represent the per protocol analysis and, given that all studies were randomised controlled studies, their original plan was also to use the intention to treat data, which many believe is the most appropriate to use for randomised controlled studies. The authors did not report the intention to treat data in the original paper as this set of data showed no difference from the per protocol analysis. Using intention to treat data, the authors found the paper by Engstrom et al had an odds ratio (95\% confidence interval) of 1.18 ( 0.75 to 1.85$)$ and the overall pooled estimate was 1.24 (0.96 to 1.6), showing no difference between treatment groups. The authors conclude that these findings, based on the intention to treat data, show that steroids plus antivirals may not be better than steroids alone, which supports the study's original conclusions.

Cite this as: BMJ 2013;346:f151

๑ BMJ Publishing Group Ltd 2013 